\author{
S. O. Gorbonos*, P. I. Kogut** \\ * Дніпропетровський національний університет ім. Олеся Гончара, \\ Дніпропетровськ 49050. E-mail: gorbonos.so@gmail.com \\ ** Дніпропетровський національний університет ім. Олеся Гончара, \\ Дніпропетровськ 49050. E-mail: p.kogut@i.ua
}

\title{
On non-variational solutions to optimal boundary control problems for parabolic equations
}

Досліджується задача оптимального керування для лінійного параболічного рівняння з необмеженими коефіцієнтами в головній частині еліптичного оператора. Особливість даного рівняння полягає в тому, що матриця потоку є кососиметричною, а її коефіцієнти належать до простору $L^{2}$. Показано, що поставлена задача керування має єдиний розв'язок, який не можна досягти через границю оптимальних розв'язків для $L^{\infty}$ - апроксимованих задач.

Ключові слова: параболічне рівняння, оптимальне керування, варіаційний роз'язок, необмежені коефіцієнти, кососиметрична матриця.

Изучается задача оптимального управления для линейного параболического уравнения с неограниченными коэффициентами в главной части эллиптического оператора. Особенность данного уравнения состоит в том, что матрица потока является кососимметричной, а ее коэффициенты принадлежат пространству $L^{2}$. Показано, что данная задача управления имеет единственное решение, которое нельзя достичь через предел оптимальных решений для $L^{\infty}$-аппроксимированных задач.

Ключевые слова: параболическое уравнение, оптимальное управление, вариационное решение, неограниченные коэффициенты, кососимметрическая матрица.

We study an optimal boundary control problem (OCP) associated to the linear parabolic equation $y_{t}-\operatorname{div}(\nabla y+A(x) \nabla y)=f$. The characteristic feature of this equation is the fact that the matrix $A(x)=\left[a_{i j}(x)\right]_{i, j=1, \ldots, N}$ is skew-symmetric, $a_{i j}(x)=-a_{j i}(x)$ and belongs to $L^{2}$-space (rather than $L^{\infty}$ ). We show that under special choice of matrix $A$ and distribution $f$, a unique solution to the original OCP inherits a singular character of the original matrix $A$ and it can not be attainable by the solutions of the similar OCPs with $L^{\infty}$-approximations of matrix $A$.

Key words: parabolic equation, optimal control, variational solution, unbounded coefficients, skew-symmetric matrix.

\section{Introduction}

We consider the optimal boundary control problem for a parabolic equation with unbounded coefficients. The characteristic feature of this problem is the fact that the stream matrix $A(x)$ is skew-symmetric and its coefficients belongs to $L^{2}$-space (rather 
than $\left.L^{\infty}\right)$. As a result, the existence, uniqueness, and variational properties of the weak solution to optimal control problem (OCP) usually are drastically different from the corresponding properties of solutions to the parabolic equations with $L^{\infty}$-matrices in coefficients. In most cases, the situation can change dramatically for the matrices $A$ with unremovable singularity. Typically, in such cases, boundary value problem may admit infinitely many weak solutions which can be divided into two classes: approximable and non-approximable solutions [5], [12], and [13].

The aim of this work is to consider OCP with a well prescribed skew-symmetric $L^{2}$ matrix $A$ and, using the direct method in the Calculus of variations, to show that this problem admits a unique solution possessing a special singular properties. As a result, we prove that this solution cannot be attained through a sequence of optimal solutions to regularized OCP for boundary value problem with skew-symmetric matrices $A_{k} \in$ $L^{\infty}\left(\Omega ; \mathbb{S}^{3}\right)$ such that $A_{k} \rightarrow A$ strongly in $L^{2}\left(\Omega ; \mathbb{S}^{3}\right)$. Thus, this result shows that a numerical analysis of optimal control problems for parabolic equations with unbounded coefficients is a non-trivial matter and it requires the elaboration of special approaches.

\section{Notation and Preliminaries}

Let $\Omega$ be the unit ball in $\mathbb{R}^{3}, \Omega=\left\{x \in \mathbb{R}^{3}:\|x\|_{\mathbb{R}^{3}}<1\right\}$. Let $C_{0}^{\infty}\left(\Omega ; \Gamma_{D}\right)$ be the set of all infinitely differentiable functions $\varphi: \Omega \rightarrow \mathbb{R}$ with compact supports in $\Omega$. Let $C_{0}^{\infty}\left(\mathbb{R}^{N} ; \Gamma_{D}\right)=\left\{\varphi \in C_{0}^{\infty}\left(\mathbb{R}^{N}\right): \varphi=0\right.$ on $\left.\Gamma_{D}\right\}$. We define the Banach space $H_{0}^{1}\left(\Omega ; \Gamma_{D}\right)$ as the closure of $C_{0}^{\infty}\left(\Omega ; \Gamma_{D}\right)$ with respect to the norm (see [1])

$$
\|y\|_{H_{0}^{1}\left(\Omega ; \Gamma_{D}\right)}=\left(\int_{\Omega}\|\nabla y\|_{\mathbb{R}^{3}}^{2} d x\right)^{1 / 2} .
$$

Let $H^{-1}\left(\Omega ; \Gamma_{D}\right)$ be the dual space to $H_{0}^{1}\left(\Omega ; \Gamma_{D}\right)$.

Let $\mathbf{X}$ be a Banach space and let $T>0$ be a given value. We denote by $L^{2}(0, T ; \mathbf{X})$ the set of measurable functions $y \in(0, T) \rightarrow \mathbf{X}$ such that $\|u(\cdot)\|_{\mathbf{X}} \in L^{2}(0, T)$. Similarly, one can also define the set of distributions $\mathcal{D}^{\prime}(0, T ; \mathbf{X})$ on $(0, T)$ with values in $\mathbf{X}$. $L^{2}(0, T ; \mathbf{X})$ is a Banach space with respect to the norm

$$
\|y\|_{L^{2}(0, T ; \mathbf{X})}=\left(\int_{0}^{T}\|u(x)\|_{\mathbf{X}}^{2} d x\right)^{1 / 2} .
$$

If $\mathbf{X}$ is reflexive, the space $L^{2}(0, T ; \mathbf{X})$ is reflexive too. Moreover, if $\mathbf{X}$ is separable, then $L^{2}(0, T ; \mathbf{X})$ is separable.

Let $C\left([0, T] ; L^{2}(\Omega)\right)$ be the space of measurable functions on $[0, T] \times \Omega$ such that $y(t, \cdot) \in L^{2}(\Omega)$ for any $t \in[0, T]$ and such that the map $t \in[0, T] \mapsto y(t, \cdot) \in L^{2}(\Omega)$ is continuous. Let us define the Banach space

$$
\mathcal{W}_{\Gamma_{D}}=\left\{y: y \in L^{2}\left(0, T ; H_{0}^{1}\left(\Omega ; \Gamma_{D}\right)\right), \frac{\partial y}{\partial t} \in L^{2}\left(0, T ; H^{-1}\left(\Omega ; \Gamma_{D}\right)\right)\right\},
$$

equipped with the norm of the graph. Here, the derivative $\partial y / \partial t$ is the distribution in $\mathcal{D}^{\prime}\left(0, T ; H^{-1}\left(\Omega ; \Gamma_{D}\right)\right)$. Then the following properties holds true (see $\left.[4,10]\right)$. 
Theorem 1. (1) The embedding $\mathcal{W}_{\Gamma_{D}} \hookrightarrow L^{2}\left(0, T ; L^{2}(\Omega)\right)$ is compact.

(2) One has the embedding $\mathcal{W}_{\Gamma_{D}} \hookrightarrow C\left([0, T] ; L^{2}(\Omega)\right)$.

(3) For any $u, v \in \mathcal{W}_{\Gamma_{D}}$, one has

$$
\begin{aligned}
\frac{d}{d t} \int_{\Omega} u(t, x) v(t, x) d x & =\left\langle u^{\prime}(t, \cdot), v(t, \cdot)\right\rangle_{H^{-1}\left(\Omega ; \Gamma_{D}\right), H_{0}^{1}\left(\Omega ; \Gamma_{D}\right)} \\
& +\left\langle v^{\prime}(t, \cdot), u(t, \cdot)\right\rangle_{H^{-1}\left(\Omega ; \Gamma_{D}\right), H_{0}^{1}\left(\Omega ; \Gamma_{D}\right)} .
\end{aligned}
$$

Let $y \in L^{2}\left(0, T ; H_{0}^{1}\left(\Omega ; \Gamma_{D}\right)\right) \cap C\left([0, T] ; L^{2}(\Omega)\right)$. Then the following density result holds: there exists $\Phi \in C^{\infty}\left([0, T] ; C_{0}^{\infty}\left(\Omega ; \Gamma_{D}\right)\right)$ such that

$$
\|y-\Phi\|_{C\left([0, T] ; L^{2}(\Omega)\right)} \leq \delta, \quad\|\nabla y-\nabla \Phi\|_{L^{2}\left(0, T ; L^{2}(\Omega)\right)} \leq \delta, \forall \delta>0 .
$$

Skew-Symmetric Matrices. Let $\mathbb{S}^{3}$ be the set of all skew-symmetric matrices $A=$ $\left[a_{i j}\right]_{i, j=1}^{3}$, i.e., $A$ is a square matrix with $a_{i j}=-a_{j i}$ and, hence, $a_{i i}=0$. Therefore, the set $\mathbb{S}^{3}$ can be identified with the Euclidean space $\mathbb{R}^{3}$.

Let $L^{2}\left(\Omega ; \mathbb{S}^{3}\right)$ be the space of measurable square-integrable functions whose values are skew-symmetric matrices and it is endowed with the norm

$$
\|A\|_{L^{2}\left(\Omega ; \mathbb{S}^{3}\right)}=\left(\int_{\Omega}\|A(x)\|_{\mathbb{S}^{3}}^{2} d x\right)^{1 / 2} .
$$

In what follows, we associate with matrix $A \in L^{2}\left(\Omega ; \mathbb{S}^{3}\right)$ the bilinear form $\varphi(\cdot, \cdot)_{A}$ : $L^{2}\left(0, T ; C_{0}^{1}(\Omega)\right) \times L^{2}\left(0, T ; C_{0}^{1}(\Omega)\right) \rightarrow \mathbb{R}$ following the rule

$$
\varphi(y, v)_{A}=\int_{0}^{T} \int_{\Omega}(\nabla v, A(x) \nabla y)_{\mathbb{R}^{3}} d x d t, \quad \forall y, v \in L^{2}\left(0, T ; C_{0}^{1}(\Omega)\right) .
$$

It is easy to see that this form is unbounded on $L^{2}\left(0, T ; H_{0}^{1}(\Omega)\right)$, since, in general, the 'integrand' $(\nabla v, A(x) \nabla y)_{\mathbb{R}^{3}}$ is not integrable on $(0, T) \times \Omega$. This motivates an introduction of the following set. We say that a distribution $y \in L^{2}\left(0, T ; H_{0}^{1}\left(\Omega ; \Gamma_{D}\right)\right)$ belongs to the set $D(A)$ if

$$
\left|\int_{0}^{T} \int_{\Omega}(\nabla \varphi, A \nabla y)_{\mathbb{R}^{3}} d x d t\right| \leq c(y, A)\left(\int_{0}^{T} \int_{\Omega}\|\nabla \varphi\|_{\mathbb{R}^{3}}^{2} d x d t\right)^{1 / 2}
$$

for all $\varphi \in C^{\infty}\left([0, T] ; C_{0}^{\infty}\left(\Omega ; \Gamma_{D}\right)\right)$, with some constant $c$ depending on $y$ and $A$. As a result, having set

$$
[y, \varphi]=\int_{0}^{T} \int_{\Omega}(\nabla \varphi, A \nabla y)_{\mathbb{R}^{N}} d x d t, \forall y \in D(A), \forall \varphi \in C^{\infty}\left([0, T] ; C_{0}^{\infty}(\Omega)\right),
$$


we observe that the bilinear form $[y, \varphi]$ can be defined for all $\varphi \in L^{2}\left(0, T ; H_{0}^{1}\left(\Omega ; \Gamma_{D}\right)\right)$ using the standard rule

$$
[y, \varphi]=\lim _{\rightarrow \infty}\left[y, \varphi_{\varepsilon}\right]
$$

where $\left\{\varphi_{\varepsilon}\right\}_{\varepsilon>0} \subset C^{\infty}\left([0, T] ; C_{0}^{\infty}\left(\Omega ; \Gamma_{D}\right)\right)$ and $\varphi_{\varepsilon} \rightarrow \varphi$ converges strongly in $L^{2}\left(0, T ; H_{0}^{1}\left(\Omega ; \Gamma_{D}\right)\right)$. In this case the value $[y, \varphi]$ is finite for every $y \in D(A)$, although the 'integrand' $(\nabla \varphi, A(x) \nabla y)_{\mathbb{R}^{3}}$ need not be integrable on $(0, T) \times \Omega$, in general. This fact leads us to the conclusion

$$
|[y, y]|<+\infty, \quad \forall y \in D(A) .
$$

At the same time, if we temporary assume that $A \in L^{\infty}\left(\Omega ; \mathbb{S}^{3}\right)$, then the bilinear form $[y, \varphi]$ is obviously bounded on $L^{2}\left(0, T ; H_{0}^{1}\left(\Omega ; \Gamma_{D}\right)\right)$, i.e. in this case $D(A) \equiv$ $L^{2}\left(0, T ; H_{0}^{1}\left(\Omega ; \Gamma_{D}\right)\right)$. Indeed, in view of the Bunjakowski inequality, we get

$$
\begin{aligned}
|[y, v]| & \leq\|A\|_{L^{\infty}\left(\Omega ; \mathbb{S}^{3}\right)} \int_{0}^{T} \int_{\Omega}\|\nabla y\|_{\mathbb{R}^{3}}\|\nabla v\|_{\mathbb{R}^{3}} d x d t \\
& \leq\|A\|_{L^{\infty}\left(\Omega ; \mathbb{S}^{3}\right)}\|y\|_{L^{2}\left(0, T ; H_{0}^{1}\left(\Omega ; \Gamma_{D}\right)\right)}\|v\|_{L^{2}\left(0, T ; H_{0}^{1}\left(\Omega ; \Gamma_{D}\right)\right)}
\end{aligned}
$$

Moreover, if $y=v$ then $[y, y]=-[y, y]$, and, therefore, $[y, y]=0$ for all $y \in$ $L^{2}\left(0, T ; H_{0}^{1}\left(\Omega ; \Gamma_{D}\right)\right)$. However, as it is shown in the next section, there exist skewsymmetric $L^{2}$-matrices $A$ such that the equality $|[y, y]|=[y, y]$ does not hold true for some $y \in D(A)$.

We define the divergence $\operatorname{div} A$ of a skew-symmetric matrix $A \in L^{2}\left(\Omega ; \mathbb{S}^{3}\right)$ as a vector-valued distribution $d \in H^{-1}\left(\Omega ; \mathbb{R}^{3}\right)$ by the following rule

$$
\left\langle d_{i}, \varphi\right\rangle_{H^{-1}(\Omega) ; H_{0}^{1}(\Omega)}=-\int_{\Omega}\left(a_{i}, \nabla \varphi\right)_{\mathbb{R}^{3}} d x, \forall \varphi \in C_{0}^{\infty}(\Omega),
$$

where $a_{i}$ stands for the $i$-th row of the matrix $A$. We say that a matrix $A \in L^{2}\left(\Omega ; \mathbb{S}^{3}\right)$ belongs to the space $H\left(\Omega, \operatorname{div} ; \mathbb{S}^{3}\right)$ if $d:=\operatorname{div} A \in L^{1}\left(\Omega ; \mathbb{R}^{3}\right)$, that is,

$$
H\left(\Omega, \operatorname{div} ; \mathbb{S}^{3}\right)=\left\{A \mid A \in L^{2}\left(\Omega ; \mathbb{S}^{3}\right), \operatorname{div} A \in L^{1}\left(\Omega ; \mathbb{R}^{3}\right)\right\}
$$

\section{Setting and Approximation of the Optimal Control Problem}

We deal with the following optimal control problem (OCP) for a parabolic equation with unbounded coefficients

$$
\begin{gathered}
I(u, y)=\left\|y-y_{d}\right\|_{L^{2}\left(0, T ; H_{0}^{1}\left(\Omega ; \Gamma_{D}\right)\right)}^{2}+\left\|u-u_{d}\right\|_{L^{2}\left(0, T ; L^{2}\left(\Gamma_{N}\right)\right)}^{2} \rightarrow \inf \\
\text { subject to the constraints } \\
y_{t}-\operatorname{div}(\nabla y+A(x) \nabla y)=f \quad \text { in }(0, T) \times \Omega, \\
y(0, \cdot)=y_{0} \quad \text { in } \Omega, \\
y(\cdot, x)=0 \quad \text { on }(0, T) \times \Gamma_{D}, \quad \frac{\partial y(\cdot, x)}{\partial \nu_{A}}=u \quad \text { on }(0, T) \times \Gamma_{N}, \\
u \in L^{2}\left(0, T ; L^{2}\left(\Gamma_{N}\right)\right),
\end{gathered}
$$


where $\Omega$ be the unit ball in $\mathbb{R}^{3}$ and its boundary $\Gamma=\left\{\|x\|_{\mathbb{R}^{3}}=1\right\}$ is divided onto two disjoint parts $\partial \Omega=\Gamma_{D} \cup \Gamma_{N}$ which have positive 2-dimensional measures. Here, $u$ is a control, $y_{0} \in L^{2}(\Omega), y_{d} \in L^{2}\left(0, T ; H_{0}^{1}(\Omega)\right)$ and $u_{d} \in L^{2}\left(0, T ; L^{2}\left(\Gamma_{N}\right)\right)$ and $f \in$ $L^{2}\left(0, T ; H^{-1}\left(\Omega ; \Gamma_{D}\right)\right)$ are given distributions, and $A \in L^{2}\left(\Omega ; \mathbb{S}^{3}\right)$ is a skew-symmetric matrix.

The optimal control problem which we consider is to minimize the discrepancy (tracking error) between a given distribution $y_{d} \in L^{2}\left(0, T ; H_{0}^{1}(\Omega)\right)$ and a solution $y$ of the Neumann-Dirichlet boundary value problem for parabolic equation (7)-(9) by choosing an appropriate boundary control $u \in L^{2}\left(0, T ; L^{2}\left(\Gamma_{N}\right)\right)$, where

$$
\frac{\partial y}{\partial \nu_{A}}=\sum_{i, j=1}^{3}\left(\delta_{i j}+a_{i j}(x)\right) \frac{\partial y}{\partial x_{j}} \cos \left(\nu, x_{i}\right),
$$

$\delta_{i j}$ is the Kronecker's delta, $\cos \left(\nu, x_{i}\right)$ is the $i$-th directing cosine of $\nu$, and $\nu$ is the outward unit normal vector at $\Gamma_{N}$ to the ball $\Omega$.

More precisely, we are concerned with OCP (6)-(10). The distinguishing feature of this problem is the special choice of matrix $A$ and distribution $f$. This entails a number of pathologies with respect to the standard properties of optimal control problems for parabolic equation and leads to the non-uniqueness of weak solutions to the corresponding initial boundary value problem and a singular properties of an optimal pair. As a result, numerical approximation of the solution to OCP (6)-(10) is getting non-trivial.

Note that the function $y=y(u)$ is called an approximable solution to the initialboundary value problem in (7)-(9) if it can be attained by weak solutions to the similar boundary value problems with $L^{\infty}$-approximated matrix $A$. However, this type of solutions does not exhaust all weak solutions to the above problem. There is another type of weak solutions, which cannot be approximated by weak solutions of such regularized problems. Usually, such solutions are called non-variational [7, 12, 13], singular [14], [2], [8], pathological [9], [11] and others

To begin with, we introduce the following notion.

Definition 1. We say that $(u, y)$ is an admissible pair to OCP (6)-(10) if $u \in$ $L^{2}\left(0, T ; L^{2}\left(\Gamma_{N}\right)\right), y \in \mathcal{W}$,

$$
y(0, \cdot)=y_{0} \in L^{2}(\Omega) \text { almost everywhere in } \Omega,
$$

and the integral identity

$$
\begin{aligned}
\int_{0}^{T} & \int_{\Omega} y_{t} \varphi d x d t+\int_{0}^{T} \int_{\Omega}(\nabla \varphi, \nabla y+A(x) \nabla y)_{\mathbb{R}^{N}} d x d t \\
& =\int_{0}^{T}\langle f, \varphi\rangle_{H^{-1}\left(\Omega ; \Gamma_{D}\right) ; H_{0}^{1}\left(\Omega ; \Gamma_{D}\right)} d t+\int_{0}^{T} \int_{\Gamma_{N}} u \varphi d \mathcal{H}^{2} d t
\end{aligned}
$$

holds true for each $\varphi \in C^{\infty}\left([0, T] ; C_{0}^{\infty}\left(\Omega ; \Gamma_{D}\right)\right)$.

We denote by $\Xi$ the set of all admissible pairs for the OCP (6)-(10). 
It is worth to note that in view of definition of the space $\mathcal{W}$ and Theorem 1 , the condition (11) has a sense. Moreover, as was shown in [3], if $(u, y)$ is an admissible pair, then $y \in D(A)$.

Definition 2. We say that OCP (6)-(10) is regular if it admits at least one admissible pair, i.e. $\Xi \neq \emptyset$.

We also say that a pair $\left(u^{0}, y^{0}\right) \in L^{2}\left(0, T ; L^{2}\left(\Gamma_{N}\right)\right) \times D(A)$ is optimal for problem

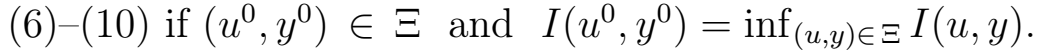

As immediately follows from (12) and the definition of bilinear form $[y, \varphi]$ (see also the extension rule $(3)$ ), every admissible pair $(u, y) \in \Xi$ is related by the following energy equality

$$
\begin{aligned}
& \frac{1}{2} \int_{0}^{T} \int_{\Omega}\left(y^{2}\right)_{t} d x d t+\|y\|_{L^{2}\left(0, T ; H_{0}^{1}\left(\Omega ; \Gamma_{D}\right)\right)}^{2}+[y, y] \\
& \quad=\int_{0}^{T}\langle f, y\rangle_{H^{-1}\left(\Omega ; \Gamma_{D}\right) ; H_{0}^{1}\left(\Omega ; \Gamma_{D}\right)} d t+\int_{0}^{T} \int_{\Gamma_{N}} u y d \mathcal{H}^{2} d t
\end{aligned}
$$

The next question which we are going to discuss is about variational solutions to the problem (6)-(10). Since $A \in L^{2}\left(\Omega ; \mathbb{S}^{3}\right)$, it follows that there exists a sequence of skewsymmetric matrices $\left\{A_{k}\right\}_{k \in \mathbb{N}} \subset L^{\infty}\left(\Omega ; \mathbb{S}^{3}\right)$ such that $A_{k} \rightarrow A$ strongly in $L^{2}\left(\Omega ; \mathbb{S}^{3}\right)$. Hence, it is reasonably, from numerical point of view, to consider the following sequence of constrained minimization problems associated with matrices $A_{k}$.

$$
\left\{\left\langle\inf _{(u, y) \in \Xi_{k}} I_{k}(u, y)\right\rangle, \quad k \rightarrow \infty\right\} .
$$

Here,

$$
I_{k}(u, y):=I(u, y)
$$

for every $\left.(u, y) \in L^{2}\left(0, T ; L^{2}\left(\Gamma_{N}\right)\right) \times L^{2}\left(0, T ; H_{0}^{1}\left(\Omega ; \Gamma_{N}\right)\right)\right), \forall k \in \mathbb{N}$ and $(u, y) \in \Xi_{k}$ if and only if

$$
\left\{\begin{array}{c}
y_{t}-\operatorname{div}\left(\nabla y+A_{k} \nabla y\right)=f \quad \text { in }(0, T) \times \Omega, \\
y(0, \cdot)=y_{0} \quad \text { in } \Omega \\
y(\cdot, x)=0 \text { on }(0, T) \times \Gamma_{D}, \frac{\partial y(\cdot, x)}{\partial \nu_{A_{k}}}=u \text { on }(0, T) \times \Gamma_{N}, \\
u \in L^{2}\left(0, T ; L^{2}\left(\Gamma_{N}\right)\right), y \in L^{2}\left(0, T ; H_{0}^{1}\left(\Omega ; \Gamma_{D}\right)\right) \\
y_{t} \in L^{2}\left(0, T ; H^{-1}\left(\Omega ; \Gamma_{D}\right)\right) .
\end{array}\right\}
$$

Theorem 2. Let $u_{d} \in L^{2}\left(0, T ; L^{2}\left(\Gamma_{N}\right)\right), f \in L^{2}\left(0, T ; H^{-1}\left(\Omega ; \Gamma_{D}\right)\right), y_{0} \in L^{2}(\Omega)$, and $y_{d} \in L^{2}\left(0, T ; H_{0}^{1}\left(\Omega ; \Gamma_{D}\right)\right)$ be given distributions. Then for every $k \in \mathbb{N}$ there exists a unique minimizer $\left(u_{k}^{0}, y_{k}^{0}\right) \in \Xi_{k}$ to the corresponding constrained minimization problem (14) such that the sequence of optimal pairs $\left\{\left(u_{k}^{0}, y_{k}^{0}\right) \in \Xi_{k}\right\}_{k \in \mathbb{N}}$ is relatively compact with 
respect to the product of the weak topologies on $L^{2}\left(0, T ; L^{2}\left(\Gamma_{N}\right)\right) \times L^{2}\left(0, T ; H_{0}^{1}\left(\Omega ; \Gamma_{D}\right)\right)$ and each of its cluster pairs $\left(u^{*}, y^{*}\right)$ possesses the properties:

$$
\left(u^{*}, y^{*}\right) \in \Xi, \quad\left[y^{*}, y^{*}\right] \geq 0 .
$$

Proof. To begin with, we show that the sequence of minimal values for the problems (14) is uniformly bounded, i.e.

$$
\sup _{k \in \mathbb{N}} \inf _{(u, y) \in \Xi_{k}} I_{k}(u, y) \leq C \text { for some } C>0 .
$$

Indeed, for every $k \in \mathbb{N}$ the bilinear form $[y, \varphi]_{k}:=\int_{0}^{T} \int_{\Omega}\left(\nabla \varphi, A_{k}(x) \nabla y\right)_{\mathbb{R}^{N}} d x d t$ is obviously bounded on $L^{2}\left(0, T ; H_{0}^{1}\left(\Omega ; \Gamma_{D}\right)\right)$. Moreover, since

$$
\int_{0}^{T} \int_{\Omega}\left(\nabla \varphi, A_{k} \nabla y\right)_{\mathbb{R}^{N}} d x d t=-\int_{0}^{T} \int_{\Omega}\left(\nabla y, A_{k} \nabla \varphi\right)_{\mathbb{R}^{N}} d x d t
$$

we have

$$
\int_{0}^{T} \int_{\Omega}\left(\nabla v, A_{k}(x) \nabla v\right)_{\mathbb{R}^{N}} d x d t=0 \quad \forall v \in L^{2}\left(0, T ; H_{0}^{1}\left(\Omega ; \Gamma_{D}\right)\right)
$$

and, hence, the initial-boundary value problem (16) has a unique solution (see [10] for the details)

$$
y_{k} \in L^{2}\left(0, T ; H_{0}^{1}\left(\Omega ; \Gamma_{D}\right)\right), \quad\left(y_{k}\right)_{t} \in L^{2}\left(0, T ; H^{-1}\left(\Omega ; \Gamma_{D}\right)\right)
$$

for every $u \in L^{2}\left(0, T ; L^{2}\left(\Gamma_{N}\right)\right)$.

As an obvious consequence of this observation and the properties of lower semicontinuity and strict convexity of the cost functional $I_{k}$, we have: the corresponding minimization problem (14) admits a unique solution [6]

$$
I_{k}\left(u_{k}^{0}, y_{k}^{0}\right)=\inf _{(u, y) \in \Xi_{k}} I_{k}(u, y), \quad\left(u_{k}^{0}, y_{k}^{0}\right) \in \Xi_{k}
$$

Moreover, having fixed a control $u \in L^{2}\left(0, T ; L^{2}\left(\Gamma_{N}\right)\right)$, condition (19) implies the fulfilment of the following identities for every $k \in \mathbb{N}$

$$
\begin{aligned}
\int_{0}^{T} \int_{\Omega}\left(y_{k}\right)_{t} \varphi d x d t+\int_{0}^{T} \int_{\Omega} & \left(\nabla \varphi, \nabla y+A_{k}(x) \nabla y_{k}\right)_{\mathbb{R}^{N}} d x d t \\
= & \int_{0}^{T}\langle f, \varphi\rangle_{H^{-1}\left(\Omega ; \Gamma_{D}\right) ; H_{0}^{1}\left(\Omega ; \Gamma_{D}\right)} d t+\int_{0}^{T} \int_{\Gamma_{N}} u \varphi d \mathcal{H}^{2} d t, \\
\frac{1}{2}\left\|y_{k}(\tau, \cdot)\right\|_{L^{2}(\Omega)}^{2}+\left\|y_{k}\right\|_{L^{2}\left(0, T ; H_{0}^{1}\left(\Omega ; \Gamma_{D}\right)\right)}^{2} & =\int_{0}^{\tau}\left\langle f, y_{k}\right\rangle_{H^{-1}\left(\Omega ; \Gamma_{D}\right) ; H_{0}^{1}\left(\Omega ; \Gamma_{D}\right)} d t \\
& \quad \int_{0}^{\tau} \int_{\Gamma_{N}} u y_{k} d \mathcal{H}^{2} d t+\frac{1}{2}\left\|y_{0}\right\|_{L^{2}(\Omega)}^{2}, \quad \forall \tau \in[0, T],
\end{aligned}
$$


where $y_{k}=y_{k}(u) \in L^{2}\left(0, T ; H_{0}^{1}(\Omega)\right)$ are the corresponding solutions to the initialboundary value problems (16). Hence, following the standard technique [10], it is easy to show that the sequence $\left\{y_{k}\right\}_{k \in \mathbb{N}}$ is bounded in $\mathcal{W}_{\Gamma_{D}}$ for every fixed $u \in L^{2}\left(0, T ; L^{2}\left(\Gamma_{N}\right)\right)$ and due to the a priori estimates

$$
\begin{aligned}
\left\|y_{k}\right\|_{L^{2}\left(0, T ; H_{0}^{1}\left(\Omega ; \Gamma_{D}\right)\right)} & +\left\|\frac{\partial y_{k}}{\partial t}\right\|_{L^{2}\left(0, T ; H^{-1}\left(\Omega ; \Gamma_{D}\right)\right)} \\
& \leq C(\Omega)\left[\|f\|_{L^{2}\left(0, T ; H^{-1}\left(\Omega ; \Gamma_{D}\right)\right)}+\|u\|_{L^{2}\left(0, T ; L^{2}\left(\Gamma_{N}\right)\right)}+\left\|y_{0}\right\|_{L^{2}(\Omega)}\right]
\end{aligned}
$$

where the constant $C(\Omega)$ is independent of $A_{k}$, we arrive at the relation

$$
\begin{aligned}
I_{k}\left(u_{k}^{0}, y_{k}^{0}\right)= & \inf _{(u, y) \in \Xi_{k}} I_{k}(u, y) \leq I_{k}\left(u, y_{k}\right) \leq 2\left\|y_{d}\right\|_{L^{2}\left(0, T ; H_{0}^{1}\left(\Omega ; \Gamma_{D}\right)\right)}^{2} \\
+2\left\|u_{d}\right\|_{L^{2}\left(0, T ; L^{2}\left(\Gamma_{N}\right)\right)}^{2} & +4 C^{2}(\Omega)\left[\|f\|_{L^{2}\left(0, T ; H^{-1}\left(\Omega ; \Gamma_{D}\right)\right)}^{2}+\left\|y_{0}\right\|_{L^{2}(\Omega)}\right] \\
& +\left(4 C^{2}(\Omega)+1\right)\|u\|_{L^{2}\left(0, T ; L^{2}\left(\Gamma_{N}\right)\right)}^{2} \leq C \quad \forall k \in \mathbb{N} .
\end{aligned}
$$

Thus, (18) holds true and it implies that

$$
\sup _{k \in \mathbb{N}}\left[\left\|y_{k}^{0}\right\|_{L^{2}\left(0, T ; H_{0}^{1}\left(\Omega ; \Gamma_{D}\right)\right)}^{2}+\left\|\frac{\partial y_{k}^{0}}{\partial t}\right\|_{L^{2}\left(0, T ; H^{-1}\left(\Omega ; \Gamma_{D}\right)\right)}^{2}+\left\|u_{k}^{0}\right\|_{L^{2}\left(0, T ; L^{2}\left(\Gamma_{N}\right)\right)}^{2}\right]<+\infty .
$$

So, by the completeness of $\mathcal{W}_{\Gamma_{D}}$, we can assume that there exists a pair $\left(u^{*}, y^{*}\right) \in$ $L^{2}\left(0, T ; L^{2}\left(\Gamma_{N}\right)\right) \times \mathcal{W}_{\Gamma_{D}}$ such that up to a subsequence

$$
\begin{gathered}
y_{k}^{0} \rightarrow y^{*} \quad \text { in } \quad L^{2}\left(0, T ; H_{0}^{1}\left(\Omega ; \Gamma_{D}\right)\right), \\
\frac{\partial y_{k}^{0}}{\partial t} \rightarrow \frac{\partial y^{*}}{\partial t} \quad \text { in } \quad L^{2}\left(0, T ; H^{-1}\left(\Omega ; \Gamma_{D}\right)\right), \\
u_{k}^{0} \rightarrow u^{*} \quad \text { in } \quad L^{2}\left(0, T ; L^{2}\left(\Gamma_{N}\right)\right) .
\end{gathered}
$$

Hence,

$$
y_{k}^{0} \longrightarrow y^{*} \quad \text { strongly in } L^{2}\left(0, T ; L^{2}\left(\Gamma_{N}\right)\right)
$$

by compactness of the embedding $H^{1 / 2}\left(\Gamma_{N}\right) \hookrightarrow L^{2}\left(\Gamma_{N}\right)$.

It remains to prove the properties (17). To do so, we note that due to the strong convergence $A_{k} \rightarrow A$ in $L^{2}\left(\Omega ; \mathbb{S}^{3}\right)$, we get

$$
\begin{gathered}
\left|\int_{0}^{T} \int_{\Omega}\left(\nabla \varphi, A \nabla y^{*}-A_{k} \nabla y_{k}^{0}\right)_{\mathbb{R}^{N}} d x d t\right| \leq \int_{0}^{T} \int_{\Omega}\left\|A_{k}-A\right\|_{\mathbb{S}^{N}}\left\|\nabla y_{k}^{0}\right\|_{\mathbb{R}^{N}}\|\nabla \varphi\|_{\mathbb{R}^{N}} d x d t \\
+\left|\int_{0}^{T} \int_{\Omega}\left(A \nabla \varphi, \nabla y^{*}-\nabla y_{k}^{0}\right)_{\mathbb{R}^{N}} d x d t\right| \\
\leq\|\varphi\|_{C\left([0, T] ; C^{1}(\bar{\Omega})\right)} \sup _{k \in \mathbb{N}}\left\|y_{k}^{0}\right\|_{L^{2}\left(0, T ; H_{0}^{1}\left(\Omega ; \Gamma_{D}\right)\right)}\left\|A_{k}-A\right\|_{L^{2}\left(\Omega ; \mathbb{S}^{3}\right)} \\
+\left|\int_{0}^{T} \int_{\Omega}\left(A \nabla \varphi, \nabla y^{*}-\nabla y_{k}^{0}\right)_{\mathbb{R}^{N}} d x d t\right| \longrightarrow 0 \text { as } k \rightarrow \infty
\end{gathered}
$$


for every $\varphi \in C^{\infty}\left([0, T] ; C_{0}^{\infty}(\Omega)\right)$.

Hence, $A_{k} \nabla y_{k}^{0} \stackrel{*}{\rightarrow} A \nabla y^{*}$ in $L^{1}\left(0, T ; L^{1}\left(\Omega ; \mathbb{R}^{3}\right)\right)$. It means that we can pass to the limit in integral identity (20) with $u=u_{k}^{0}$. As a result, we have: the pair $\left(u^{*}, y^{*}\right)$ is related by the integral identity (13), therefore, $y^{*}$ is a weak solution to the original boundary value problem (7)-(9) under $u=u^{*}$ in the sense of Definition 1. Thus, $\left(u^{*}, y^{*}\right) \in \Xi$. Moreover, following [3], we have $y^{*} \in D(A)$.

In order to prove the property $(17)_{2}$, we pass to the limit in the energy equality (21). Takin into account the lower semicontinuity of the norm $\|\cdot\|_{L^{2}\left(0, T ; H^{1}(\Omega)\right)}^{2}$ with respect to the weak convergence $\nabla y_{k}^{0} \rightarrow \nabla y^{*}$ in $L^{2}\left(0, T ; L^{2}\left(\Omega ; \mathbb{R}^{3}\right)\right)$, we obtain

$$
\begin{aligned}
& \frac{1}{2} \int_{0}^{T} \int_{\Omega}\left(y^{2}\right)_{t} d x d t+\|y\|_{L^{2}\left(0, T ; H_{0}^{1}\left(\Omega ; \Gamma_{D}\right)\right)}^{2} \\
& \leq\left\langle f, y^{*}\right\rangle_{H^{-1}\left(\Omega ; \Gamma_{D}\right) ; H_{0}^{1}\left(\Omega ; \Gamma_{D}\right)}+\lim _{k \rightarrow \infty} \int_{0}^{T} \int_{\Gamma_{N}} u_{k}^{0} y_{k}^{0} d \mathcal{H}^{2} d t
\end{aligned}
$$

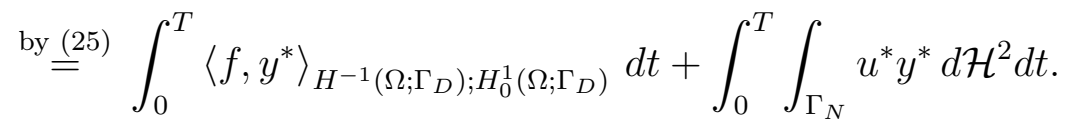

Thus, the desired inequality $(17)_{2}$ obviously follows from (13) and (26). The proof is complete.

Remark 1. As immediately follows from the proof of this theorem, some admissible pairs $\left(u^{*}, y^{*}\right) \in \Xi$ can be attained by optimal solutions to the approximate OCPs (14). Hence, we can conclude that the original optimal control problem (6)-(10) is regular for every $u_{d} \in L^{2}\left(0, T ; L^{2}\left(\Gamma_{N}\right)\right), f \in L^{2}\left(0, T ; H^{-1}\left(\Omega ; \Gamma_{D}\right)\right), y_{0} \in L^{2}(\Omega)$, and $y_{d} \in L^{2}\left(0, T ; H_{0}^{1}\left(\Omega ; \Gamma_{D}\right)\right)$.

Remark 2 . The next observation deals with the inequality $(17)_{2}$. As Theorem 2 proves, for any approximation $\left\{A_{k}\right\}_{k \in \mathbb{N}}$ of the matrix $A \in L^{2}\left(\Omega ; \mathbb{S}^{3}\right)$ with properties $\left\{A_{k}\right\}_{k \in \mathbb{N}} \subset$ $L^{\infty}\left(\Omega ; \mathbb{S}^{3}\right)$ and $A_{k} \rightarrow A$ strongly in $L^{2}\left(\Omega ; \mathbb{S}^{3}\right)$, the optimal solutions to the regularized OCPs (14)-(16) always leads us in the limit to some admissible solution $\left(u^{*}, y^{*}\right)$ of the original OCP (6)-(10). Moreover, in general, this limit pair can depend on the choice of the approximative sequence $\left\{A_{k}\right\}_{k \in \mathbb{N}}$. That's why it is reasonably to call such pairs attainable admissible solutions to OCP (6)-(10).

As we will see later on, the pair $\left(u^{*}, y^{*}\right)$ is not optimal, in general, and the pair $\left(u_{d}, y_{d}\right)$ is a unique optimal pair to OCP $(6)-(10)$. Whereas we will shown that $\left[y_{d}, y_{d}\right]=$ $-\alpha$, where $\alpha$ is a given strictly positive value, in the mean time $\left[y^{*}, y^{*}\right] \geq 0$ for any attainable pair $\left(u^{*}, y^{*}\right)$. Thus, for given $f, y_{d}, y_{0}, u_{d}$ the optimal pair $\left(u^{0}, y^{0}\right)$ to OCP $(6)-$ (10) cannot be attained through any $L^{\infty}$-approximation of the matrix $A \in L^{2}\left(\Omega ; \mathbb{S}^{3}\right)$.

\section{Example of the Non-Variational Solution}

Our aim in this section is to show that optimal control problem (6)-(10) has a unique non-variational solution. Namely, we will show that for a given positive scalar 
value $\alpha \in \mathbb{R}$ there exist a skew-symmetric matrix $A \in L^{2}\left(\Omega ; \mathbb{S}^{3}\right)$ and a function $y_{d} \in$ $L^{2}\left(0, T ; H_{0}^{1}(\Omega)\right)$ such that

$$
y_{d} \in D(A) \text { and }\left[y_{d}, y_{d}\right]=-\alpha<0,
$$

where the bilinear form $[y, v]$ is defined by (2).

We divide our analysis into several steps. At the first step we define a skewsymmetric matrix $A$ as follows

$$
A(x)=\left(\begin{array}{ccc}
0 & a(x) & 0 \\
-a(x) & 0 & -b(x) \\
0 & b(x) & 0
\end{array}\right)
$$

where $a(x)=\frac{x_{1}}{2\|x\|_{\mathbb{R}^{3}}^{2}}$ and $b(x)=\frac{x_{3}}{2\|x\|_{\mathbb{R}^{3}}^{2}}$. Since

$$
\|a\|_{L^{2}(\Omega)}^{2}=\int_{\Omega}\left(\frac{x_{1}}{2\|x\|_{\mathbb{R}^{3}}^{2}}\right)^{2} d x=\int_{0}^{1} \int_{0}^{2 \pi} \int_{0}^{\pi} \frac{\rho^{2} \cos ^{2} \varphi \sin ^{2} \psi}{4 \rho^{4}} \rho^{2} \sin \psi d \psi d \varphi d \rho<+\infty,
$$

it follows that $a \in L^{2}(\Omega)$. By analogy, it can be shown that $b \in L^{2}(\Omega)$. Moreover, it is easy to see that the skew-symmetric matrix $A$, define by (28), satisfies the property $A \in H\left(\Omega\right.$, div; $\left.\mathbb{S}^{3}\right)$, i.e. $A \in L^{2}\left(\Omega ; \mathbb{S}^{3}\right)$ and $\operatorname{div} A \in L^{1}\left(\Omega ; \mathbb{R}^{3}\right)$. Indeed, in view of the definition of the divergence $\operatorname{div} A$ of a skew-symmetric matrix, we have $\operatorname{div} A=\left[\begin{array}{l}d_{1} \\ d_{2} \\ d_{3}\end{array}\right]$, where $d_{i}=\operatorname{div} a_{i}=\frac{x_{i} x_{2}}{\|x\|_{\mathbb{R}^{3}}^{4}}$ and $a_{i}$ is $i$-th column of $A$. As a result, we get

$$
\begin{aligned}
\left\|\operatorname{div} a_{i}\right\|_{L^{1}(\Omega)} & =\int_{0}^{1} \int_{0}^{2 \pi} \int_{0}^{\pi}\left|\frac{\rho^{2} f_{i}(\varphi, \psi) \sin \varphi \sin \psi}{\rho^{4}}\right| \rho^{2} \sin \psi d \psi d \varphi d \rho \\
& <+\infty
\end{aligned}
$$

for the corresponding $f_{i}=f_{i}(\varphi, \psi)(i=1,2,3)$. Therefore, $\operatorname{div} A \in L^{1}\left(\Omega ; \mathbb{R}^{3}\right)$.

Step 2 deals with the choice of the function $y_{d} \in L^{2}\left(0, T ; H_{0}^{1}(\Omega)\right)$. We define it by the rule

$$
\begin{aligned}
y_{d}(t, x)=t \sqrt{\frac{52 \alpha}{\pi T^{3}(1-\exp (-2 \pi))}}\left(1-\|x\|_{\mathbb{R}^{3}}^{5}\right) \\
\times \frac{x_{2}^{2}}{x_{1}^{2}+x_{2}^{2}} \exp \left(-\frac{\pi}{2}-\arctan \frac{\sqrt{x_{1}^{2}+x_{2}^{2}}-x_{1}}{x_{2}}\right),
\end{aligned}
$$

for all $(t, x) \in(0, T) \times \Omega$. It is easy to see that

$$
\begin{aligned}
v_{0}\left(\frac{x}{\|x\|_{\mathbb{R}^{3}}}\right) & :=\sqrt{\frac{52 \alpha}{\pi T^{3}(1-\exp (-2 \pi))}} \frac{x_{2}^{2}}{x_{1}^{2}+x_{2}^{2}} \exp \left(-\frac{\pi}{2}-\arctan \frac{\sqrt{x_{1}^{2}+x_{2}^{2}}-x_{1}}{x_{2}}\right) \\
& =\sqrt{\frac{52 \alpha}{\pi T^{3}(1-\exp (-2 \pi))}} \sin ^{2} \varphi \exp (-\varphi / 2), \quad \forall \varphi \in[0,2 \pi]
\end{aligned}
$$


with respect to the spherical coordinates. Hence, $v_{0} \in C^{1}(\partial \Omega)$, and, as immediately follows from (29), it provides that

$$
y_{d} \in L^{2}\left(0, T ; L^{2}(\Omega)\right) \quad \text { and } \quad y_{d}(t, \cdot)=0 \text { on } \partial \Omega \quad \forall t \in[0, T] .
$$

By direct computations, we get

$$
\nabla v_{0}\left(\frac{x}{\|x\|_{\mathbb{R}^{3}}}\right)=\frac{1}{\|x\|_{\mathbb{R}^{3}}^{3}}\left[\begin{array}{c}
\frac{\partial v_{0}}{\partial z_{1}}\left(\|x\|_{\mathbb{R}^{3}}^{2}-x_{1}^{2}\right)-\frac{\partial v_{0}}{\partial z_{2}} x_{1} x_{2} \\
\frac{\partial v_{0}}{\partial z_{2}}\left(\|x\|_{\mathbb{R}^{3}}^{2}-x_{2}^{2}\right)-\frac{\partial v_{0}}{\partial z_{1}} x_{1} x_{2} \\
-\frac{\partial v_{0}}{\partial z_{1}} x_{1} x_{3}-\frac{\partial v_{0}}{\partial z_{2}} x_{2} x_{3}
\end{array}\right], \forall x \neq 0 .
$$

Hence, there exists a constant $C^{*}>0$ such that

$$
\left\|\nabla v_{0}\left(\frac{x}{\|x\|_{\mathbb{R}^{3}}}\right)\right\|_{\mathbb{R}^{3}} \leq \frac{C^{*}}{\|x\|_{\mathbb{R}^{3}}}
$$

Thus,

$$
\begin{aligned}
\left\|\nabla y_{d}\right\|_{\mathbb{R}^{3}} \leq & t\left|v_{0}\left(\frac{x}{\|x\|_{\mathbb{R}^{3}}}\right)\right|\left\|\nabla\left(1-\|x\|_{\mathbb{R}^{3}}^{5}\right)\right\|_{\mathbb{R}^{3}} \\
& +t\left(1-\|x\|_{\mathbb{R}^{3}}^{5}\right)\left\|\nabla v_{0}\left(\frac{x}{\|x\|_{\mathbb{R}^{3}}}\right)\right\|_{\mathbb{R}^{3}} \leq C_{1}+\frac{C_{2}}{\|x\|_{\mathbb{R}^{3}}} .
\end{aligned}
$$

As a result, we infer that $\nabla y_{d} \in L^{2}\left(0, T ; L^{2}\left(\Omega ; \mathbb{R}^{3}\right)\right)$, i.e. we finally have $y_{d} \in$ $L^{2}\left(0, T ; H_{0}^{1}(\Omega)\right)$.

Step 3. We show that the function $y_{d}$, which was introduced before, belongs to the set $D(A)$. To do so, we have to prove the estimate

$$
\left|\int_{0}^{T} \int_{\Omega}\left(\nabla \varphi, A(x) \nabla y_{d}\right)_{\mathbb{R}^{3}} d x d t\right| \leq \widetilde{C}\left(\int_{0}^{T} \int_{\Omega}|\nabla \varphi|_{\mathbb{R}^{3}}^{2} d x d t\right)^{1 / 2},
$$

for all $\varphi \in C^{\infty}\left([0, T] ; C_{0}^{\infty}(\Omega)\right)$.

To this end, we make use of the following transformations

$$
\begin{aligned}
& \int_{0}^{T} \int_{\Omega}(\nabla \varphi, A \nabla \psi)_{\mathbb{R}^{3}} d x d t=-\int_{0}^{T}\langle\operatorname{div}(A \nabla \psi), \varphi\rangle_{H^{-1}(\Omega) ; H_{0}^{1}(\Omega)} d t \\
& =\int_{0}^{T}\left\langle\operatorname{div}\left[\begin{array}{c}
\left(a_{1}\right)^{t} \nabla \psi \\
\left(a_{2}\right)^{t} \nabla \psi \\
\left(a_{3}\right)^{t} \nabla \psi
\end{array}\right], \varphi\right\rangle_{H^{-1}(\Omega) ; H_{0}^{1}(\Omega)} d t \\
& =\int_{0}^{T} \sum_{i=1}^{3}\left\langle\operatorname{div} a_{i}, \varphi \frac{\partial \psi}{\partial x_{i}}\right\rangle_{H^{-1}(\Omega) ; H_{0}^{1}(\Omega)} d t+\int_{0}^{T} \underbrace{\sum_{\Omega}^{3} \sum_{i=1}^{3} \sum_{j=1}^{3}\left(a_{i j} \frac{\partial^{2} \psi}{\partial x_{i} \partial x_{j}}\right) \varphi d x}_{\substack{=0 \\
\text { since } \\
A \in L^{2}\left(\Omega ; \mathbb{S}^{3}\right)}} d t
\end{aligned}
$$

(due to the fact that $\operatorname{div} A \in L^{1}\left(\Omega ; \mathbb{R}^{3}\right)$ )

$$
=\int_{0}^{T} \int_{\Omega}(\operatorname{div} A, \nabla \psi)_{\mathbb{R}^{3}} \varphi d x d t
$$


which are obviously true for all $\psi, \varphi \in C^{\infty}\left([0, T] ; C_{0}^{\infty}(\Omega)\right)$. Since

$$
\begin{aligned}
\left|\int_{0}^{T} \int_{\Omega}(\operatorname{div} A, \nabla \psi)_{\mathbb{R}^{3}} \varphi d x d t\right| & =\left|\int_{0}^{T} \int_{\Omega}(\nabla \varphi, A \nabla \psi)_{\mathbb{R}^{3}} d x d t\right| \\
& \leq C\|A\|_{L^{2}\left(\Omega ; \mathbb{S}_{\text {skew }}^{3}\right)}\|\psi\|_{L^{2}\left(0, T ; H_{0}^{1}(\Omega)\right)}
\end{aligned}
$$

it follows that, using the continuation principle, we can extend the previous equality with respect to $\psi$ to the following one

$$
\begin{gathered}
\int_{0}^{T} \int_{\Omega}\left(\nabla \varphi, A \nabla y_{d}\right)_{\mathbb{R}^{3}} d x d t=\int_{0}^{T} \int_{\Omega} \varphi\left(\operatorname{div} A, \nabla y_{d}\right)_{\mathbb{R}^{3}} d x d t \\
\forall \varphi \in C^{\infty}\left([0, T] ; C_{0}^{\infty}(\Omega)\right)
\end{gathered}
$$

Let us show that $\left(\operatorname{div} A, \nabla y_{d}\right)_{\mathbb{R}^{3}} \in L^{\infty}((0, T) \times \Omega)$. In this case, relation (32) implies the estimate

$$
\begin{array}{rl}
\mid \int_{0}^{T} \int_{\Omega}\left(\nabla \varphi, A \nabla y_{d}\right)_{\mathbb{R}^{3}} & d x d t\left|\leq\left\|\left(\operatorname{div} A, \nabla y_{d}\right)_{\mathbb{R}^{3}}\right\|_{L^{\infty}((0, T) \times \Omega)} \int_{0}^{T} \int_{\Omega}\right| \varphi \mid d x d t \\
\leq & \widetilde{C}\left(\int_{0}^{T} \int_{\Omega}|\nabla \varphi|_{\mathbb{R}^{N}}^{2} d x d t\right)^{1 / 2}, \forall \varphi \in C^{\infty}\left([0, T] ; C_{0}^{\infty}(\Omega)\right),
\end{array}
$$

which means that the element $y_{d}$ belongs to the set $D(A)$.

Indeed, as follows from (31), we have the equality

$$
\left(\nabla v_{0}\left(\frac{x}{\|x\|_{\mathbb{R}^{3}}}\right), \frac{x}{\|x\|_{\mathbb{R}^{3}}^{3}}\right)_{\mathbb{R}^{3}}=0
$$

Thus, the gradient of the function $\nabla v_{0}\left(\frac{x}{\|x\|_{\mathbb{R}^{3}}}\right)$ is orthogonal to the vector field $Q=$ $x /\|x\|_{\mathbb{R}^{3}}^{3}$ outside the origin. Therefore,

$$
\begin{aligned}
\left(\nabla y_{d}, \operatorname{div} A\right)_{\mathbb{R}^{3}}:= & t\left(\nabla\left[\left(1-\|x\|_{\mathbb{R}^{3}}^{5}\right) v_{0}\left(\frac{x}{\|x\|_{\mathbb{R}^{3}}}\right)\right], \frac{x}{\|x\|_{\mathbb{R}^{3}}^{3}} \frac{x_{2}}{\|x\|_{\mathbb{R}^{3}}}\right)_{\mathbb{R}^{3}} \\
= & t\left(\nabla\left(1-\|x\|_{\mathbb{R}^{3}}^{5}\right), \frac{x}{\|x\|_{\mathbb{R}^{3}}^{3}}\right)_{\mathbb{R}^{3}} v_{0}\left(\frac{x}{\|x\|_{\mathbb{R}^{3}}}\right) \frac{x_{2}}{\|x\|_{\mathbb{R}^{3}}} \\
& +t\left(1-\|x\|_{\mathbb{R}^{3}}^{5}\right)\left(\nabla v_{0}\left(\frac{x}{\|x\|_{\mathbb{R}^{3}}}\right), \frac{x}{\|x\|_{\mathbb{R}^{3}}^{3}}\right) \frac{x_{2}}{\mathbb{R}^{3}}=I_{1}+I_{2},
\end{aligned}
$$

where $I_{2}=0$ by (34). Since $\nabla\left(1-\|x\|_{\mathbb{R}^{3}}^{5}\right)=-5\|x\|_{\mathbb{R}^{3}}^{3} x, \frac{x_{2}}{\|x\|_{\mathbb{R}^{3}}}=\sin \varphi \sin \psi$ with respect to the spherical coordinates, and function $v_{0}$ is smooth, it follows that there exists a constant $C_{0}>0$ such that $\left|\left(\nabla y_{d}, \operatorname{div} A\right)_{\mathbb{R}^{3}}\right| \leq C_{0}$ almost everywhere in $(0, T) \times \Omega$. Thus,

$$
\left(\operatorname{div} A, \nabla y_{d}\right)_{\mathbb{R}^{3}} \in L^{\infty}((0, T) \times \Omega)
$$

and we have obtained the required property. 
Step 4. Using results of the previous steps, we show that the function $y_{d}$ satisfies the condition $\left[y_{d}, y_{d}\right]=-\alpha<0$. Indeed, let $\left\{\varphi_{\varepsilon}\right\}_{\varepsilon \rightarrow 0} \subset C^{\infty}\left([0, T] ; C_{0}^{\infty}(\Omega)\right)$ be a sequence such that

$$
\varphi_{\varepsilon} \rightarrow y_{d} \text { strongly in } L^{2}\left(0, T ; H_{0}^{1}(\Omega)\right) .
$$

Then by continuity, we have

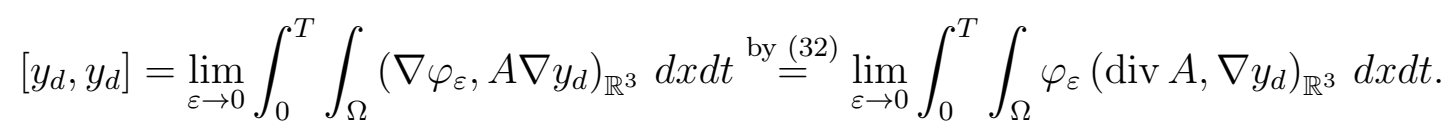

Since $\left(\operatorname{div} A, \nabla y_{d}\right)_{\mathbb{R}^{3}} \in L^{\infty}((0, T) \times \Omega)$, in view of the property (35), we can pass to the limit in the right-hand side of this relation. As a result, we get

$$
\left[y_{d}, y_{d}\right]=\int_{0}^{T} \int_{\Omega} y_{d}\left(\operatorname{div} A, \nabla y_{d}\right)_{\mathbb{R}^{3}} d x d t=\frac{1}{2} \int_{0}^{T} \int_{\Omega}\left(\operatorname{div} A, \nabla y_{d}^{2}\right)_{\mathbb{R}^{3}} d x d t
$$

Let $\Omega_{\varepsilon}=\left\{x \in \mathbb{R}^{3} \mid \varepsilon<\|x\|_{\mathbb{R}^{3}}<1\right\}$ and let $\Gamma_{\varepsilon}=\left\{\|x\|_{\mathbb{R}^{3}}=\varepsilon\right\}$ be the sphere of radius $\varepsilon$ centered at the origin. Then

$$
\begin{aligned}
\int_{0}^{T} & \int_{\Omega_{\varepsilon}}\left(\operatorname{div} A, \nabla y_{d}^{2}\right)_{\mathbb{R}^{3}} d x d t=\int_{0}^{T} \int_{\Gamma_{\varepsilon}}(\operatorname{div} A, \nu)_{\mathbb{R}^{3}} y_{d}^{2} d \mathcal{H}^{2} d t \\
& =\int_{0}^{T}\left[\int_{\Gamma_{\varepsilon}}(\operatorname{div} A, \nu)_{\mathbb{R}^{3}}\left(1-\|x\|_{\mathbb{R}^{3}}^{5}\right)^{2} v_{0}^{2}\left(\frac{x}{\|x\|_{\mathbb{R}^{3}}}\right) d \mathcal{H}^{2}\right] t^{2} d t \\
& =\frac{T^{3}}{3} \int_{\Gamma_{\varepsilon}}(\operatorname{div} A, \nu)_{\mathbb{R}^{3}} v_{0}^{2}\left(\frac{x}{\|x\|_{\mathbb{R}^{3}}}\right) d \mathcal{H}^{2}+o(1) \\
& =\frac{T^{3}}{3} \int_{\Gamma_{\varepsilon}}\left(\frac{x}{\|x\|_{\mathbb{R}^{3}}^{3}},\left(-\frac{x}{\|x\|_{\mathbb{R}^{3}}}\right)\right)_{\mathbb{R}^{3}} \frac{x_{2}}{\|x\|_{\mathbb{R}^{3}}} v_{0}^{2}\left(\frac{x}{\|x\|_{\mathbb{R}^{3}}}\right) d \mathcal{H}^{2}+o(1) \\
& =-\frac{T^{3}}{3 \varepsilon^{2}} \int_{\Gamma_{\varepsilon}} \frac{x_{2}}{\|x\|_{\mathbb{R}^{3}}} v_{0}^{2}\left(\frac{x}{\|x\|_{\mathbb{R}^{3}}}\right) d \mathcal{H}^{2}+o(1) \\
& =-\frac{T^{3}}{3} \int_{\Gamma} b_{0}(x) v_{0}^{2}(x) d \mathcal{H}^{2}+o(1),
\end{aligned}
$$

where $b_{0}=\sin \varphi \sin \psi$ and $v_{0}^{2}=\frac{52 \alpha}{\pi T^{3}(1-\exp (-2 \pi))} \sin ^{4} \varphi \exp (-\varphi)$. Since

$$
\int_{\partial \Omega} b_{0} v_{0}^{2} d \mathcal{H}^{2}=\frac{52 \alpha}{\pi T^{3}(1-\exp (-2 \pi))}\left(\int_{0}^{2 \pi} \sin ^{5} \varphi e^{-\varphi} d \varphi \int_{0}^{\pi} \sin ^{2} \psi d \psi\right)=6 \alpha T^{-3}>0
$$

it remains to combine this result with $(36),(37)$, and relation

$$
\int_{0}^{T} \int_{\Omega}\left(\operatorname{div} A, \nabla y_{d}^{2}\right)_{\mathbb{R}^{3}} d x d t=\lim _{\varepsilon \rightarrow 0} \int_{0}^{T} \int_{\Omega_{\varepsilon}}\left(\operatorname{div} A, \nabla y_{d}^{2}\right)_{\mathbb{R}^{3}} d x d t
$$

As a result, we finally infer $\left[y_{d}, y_{d}\right]=-\alpha<0$.

However, as was shown before, the value $[y, y]$ is not of constant sign on $D(A)$. Hence, energy equality (13) does not allow us to derive any a priory estimate for 
the admissible solutions. In spite of this, the following result proves that OCP (6)(10) is well-posed under the special choice of distributions $y_{d} \in L^{2}\left(0, T ; H_{0}^{1}(\Omega)\right), u_{d} \in$ $L^{2}\left(0, T ; L^{2}\left(\Gamma_{N}\right)\right), y_{0} \in L^{2}(\Omega)$, and $f \in L^{2}\left(0, T ; H^{-1}(\Omega)\right)$.

Theorem 3. Let $A \in L^{2}\left(\Omega ; \mathbb{S}^{3}\right)$ and $y_{d} \in L^{2}\left(0, T ; H_{0}^{1}(\Omega)\right)$ be defined by (28) and (29), respectively. Assume that $y_{0} \equiv 0$ in $\Omega$ and distributions $f \in L^{2}\left(0, T ; H^{-1}(\Omega)\right)$ and $u_{d} \in L^{2}\left(0, T ; L^{2}\left(\Gamma_{N}\right)\right)$ are given by the rule

$$
f:=\left(y_{d}\right)_{t}-\operatorname{div}\left(\nabla y_{d}+A \nabla y_{d}\right) \text { and } u_{d}:=\gamma_{\Gamma_{N}}^{1}\left(y_{d}\right)
$$

where $\gamma_{\Gamma_{N}}^{1}: L^{2}\left(0, T ; H_{0}^{1}\left(\Omega ; \Gamma_{D}\right)\right) \rightarrow L^{2}\left(0, T ; H^{-1 / 2}\left(\Gamma_{N}\right)\right)$ is the trace operator such that

$$
\gamma_{\Gamma_{N}}^{1}(y)=\left.\frac{\partial y}{\partial \nu_{A}}\right|_{\Gamma_{N}}:=\sum_{i, j=1}^{3}\left(\delta_{i j}+a_{i j}(x)\right) \frac{\partial y}{\partial x_{j}} \cos \left(\nu, x_{i}\right)
$$

provided $y \in L^{2}\left(0, T ; H_{0}^{1}\left(\Omega ; \Gamma_{D}\right)\right) \cap L^{2}\left(0, T ; C^{1}(\bar{\Omega})\right)$.

Then the pair $\left(u^{0}, y^{0}\right):=\left(u_{d}, y_{d}\right) \in L^{2}\left(0, T ; L^{2}\left(\Gamma_{N}\right)\right) \times D(A)$ is a unique solution to $O C P(6)-(10)$.

Proof. As follows from (29), the function $y_{d}$ is smooth near the boundary $\partial \Omega$ and $\left(y_{d}\right)_{t} \in L^{2}\left(0, T ; H_{0}^{1}(\Omega)\right)$. Hence, $u_{d}:=\gamma_{\Gamma_{N}}^{1}\left(y_{d}\right) \in L^{2}\left(\Gamma_{N}\right)$ and $y_{d} \in \mathcal{W}$ (see (30)). Moreover, the inclusion $y_{d} \in D(A)$ (see estimate (33)) implies:

$$
\operatorname{div}\left(A \nabla y_{d}\right) \in L^{2}\left(0, T ; H^{-1}(\Omega)\right) .
$$

Therefore, in view of the inclusion $\left(y_{d}\right)_{t} \in L^{2}\left(0, T ; H_{0}^{1}(\Omega)\right)$, we have $f \in$ $L^{2}\left(0, T ; H^{-1}(\Omega)\right)$. Since $y_{d}(0, \cdot)=0$ in $\Omega$ and

$$
\begin{aligned}
\int_{0}^{T} & \langle f, \varphi\rangle_{H^{-1}\left(\Omega ; \Gamma_{D}\right) ; H_{0}^{1}\left(\Omega ; \Gamma_{D}\right)} d t=\int_{0}^{T} \int_{\Omega}\left(y_{d}\right)_{t} \varphi d x d t \\
& +\int_{0}^{T} \int_{\Omega}\left(\nabla \varphi, \nabla y_{d}+A(x) \nabla y_{d}\right)_{\mathbb{R}^{N}} d x d t-\int_{0}^{T} \int_{\Gamma_{N}} \gamma_{\Gamma_{N}}^{1}\left(y_{d}\right) \varphi d \mathcal{H}^{2} d t
\end{aligned}
$$

for all $\varphi \in C^{\infty}\left([0, T] ; C_{0}^{\infty}\left(\Omega ; \Gamma_{D}\right)\right)$, it follows that the pair $\left(u_{d}, y_{d}\right)$ satisfies relations (11)-(12). Thus, $\left(u_{d}, y_{d}\right)$ is an admissible solution to OCP (6)-(10) in the sense of Definition 1. To conclude the proof, it is enough to note that

$$
I(u, y) \geq 0 \quad \forall(u, y) \in \Xi, \quad I\left(u_{d}, y_{d}\right)=0,
$$

and the cost functional $I: \Xi \rightarrow \mathbb{R}$ is strictly convex.

\section{References}

1. R. Adams, Sobolev spaces - Academic Press, New York, 1975.

2. G. Buttazzo Weak optimal controls in coefficients for linear elliptic problems/G. Buttazzo,

P. I. Kogut // Revista Matematica Complutense, 24, (2011), 83-94. 
3. S. O. Gorbonos Variational solutions of an optimal control problem with unbounded coefficient/S. O. Gorbonos, P. I. Kogut // Visnyk DNU. Series: Mathematical Modelling, Dnipropetrovsk: DNU, 5, (2013), N 8, 69-83 (in Ukrainian).

4. V. I. Ivanenko Variational Methods in Optimal Control Problems for Systems with Distributed Parameters/V. I. Ivanenko, V. S. Mel'nik - Naukova Dumka, Kyiv, 1988 (in Russian).

5. M. A. Fannjiang Diffusion in turbulence/M. A. Fannjiang, G. C. Papanicolaou // Probab. Theory and Related Fields, 105, (1996), 279-334.

6. A. V. Fursikov Optimal Control of Distributed Systems. Theory and Applications - AMS, Providence, RI, 2000.

7. P. I. Kogut On Approximation of an Optimal Boundary Control Problem for Linear Elliptic Equation with Unbounded Coefficients // Discrete and Continuous Dynamical Systems - Series A, 34, No.5, 2014, 2105-2133.

8. P. I. Kogut Optimal Control Problems for Partial Differential Equations on Reticulated Domains: Approximation and Asymptotic Analysis/P. I. Kogut, G. Leugering Birkhäuser, Boston, 2011.

9. T. Jin Pathological solutions to elliptic problems in divergence form with continuous coefficients / T. Jin, V. Mazya, J. van Schaftinger // C. R. Math. Acad. Sci. Paris, 347, (2009), N 13-14, 773-778.

10. S. Salsa, Partial Differential Equations in Action: From Modelling to Theory - SpringerVerlag, Milan, 2008.

11. J. Serrin Pathological solutions of elliptic differential equations // Ann. Scuola Norm. Sup. Pisa, 3, (1964), N 18, 385-387.

12. V. V. Zhikov Diffusion in incompressible random flow // Functional Analysis and Its Applications, 31, (1997), N 3, 156-166.

13. V. V. Zhikov Remarks on the ubiqueness of a solution of the Dirichlet problem for second-order elliptic equations with lower-order terms// Functional Analysis and Its Applications, 38, (2004), N 3, 173-183.

14. J. L. Vazquez The Hardy inequality and the asymptotic behaviour of the heat equation with an inverse-square potential / J. L. Vazquez, E. Zuazua // J. of Functional Analysis, 173, (2000), 103-153. 